\title{
Application of Lagrange Methods for Studying Dynamic Processes and Floating Objects Transport in the Ocean
}

\author{
Arseny A. Kubryakov, Sergey V. Stanichny, Eugeny I. Kalinin, Anna Yu. Kucheiko, \\ Rimma R. Stanichnaya, Alesya V. Medvedeva
}

Marine Hydrophysical Institute RAS, Sevastopol, Russia

arskubr@yandex.ru

\begin{abstract}
The system based on the integrated use of satellite altimetry data and wind speed from atmospheric reanalyses is proposed for calculation and prediction the trajectories of moving objects in the World Ocean. The proposed method makes it possible to sufficiently determine the movement of oil pollution, suspensions on the sea surface and to assess the distribution of river water in the ocean. Examples of comparing the calculated trajectories with satellite data are given. They used both sequential images, which reproduced the movement of spatial inhomogeneities at time intervals of up to several days, and an estimate of the integral distribution of river flows in the interval of one month. On the basis of the proposed method, a system for calculating the movement of floating objects FOTS (floating object tracking system) was created. To calculate the trajectory of objects, the system uses data from modern satellite and meteorological archives that are freely available on the Internet.
\end{abstract}

Keywords: Lagrange modelling, oil pollution, water pollution, surface currents, satellite monitoring, transport forecast 


\title{
Применение Лагранжевых методов для исследования динамических процессов и транспорта плавающих объектов в океане
}

\author{
А.А Кубряков, С.В Станичный, Е.И. Калинин, А.Ю. Кучейко, Р.Р. Станичная, \\ A.В. Медведева
}

Морской гидрофизический институт РАН, Севастополь, Россия arskubr@yandex.ru

\begin{abstract}
В работе на основе комплексного использования спутниковых альтиметрических данных и скорости ветра из атмосферных реанализов предлагается система, позволяющая рассчитывать и прогнозировать траектории перемещения плавающих объектов на акватории всего Мирового Океана. Предложенный метод позволяет достаточно хорошо определять перемещение нефтяных загрязнений, взвесей на морской поверхности и оценивать распространение речных вод в океане. Приведены примеры сопоставления расчётных траекторий со спутниковыми данными. Использованы как последовательные изображения, на которых воспроизводилось движение пространственных неоднородностей на временных интервалах до нескольких суток, так и оценка интегрального распространения речных стоков на интервале один месяц. На основе предложенного метода была создана система расчета перемещения плавающих объектов FOTS (floating object tracking system). Для расчета траектории объектов система использует современные спутниковые и метеорологические архивы данных, находящихся в свободном доступе в интернете.
\end{abstract}

Ключевые слова: моделирование траекторий лагранжевых частиц, нефтяные загрязнения, загрязнение вод, поверхностные течения, спутниковый мониторинг, прогноз распространения

\section{Введение}

Расчет траекторий плавающих объектов на морской поверхности необходим для решения большого количества важных прикладных задач. К ним относятся, например, определение переноса нефтяных или иных загрязняющих веществ в океане; расчет переноса обломков судов или самолетов после корабле- и авиакатастроф и поиск экипажа после аварий на воде; определение направления движения айсбергов и плавающих льдин, представляющих опасность для судоходства. Кроме того, они могут быть использованы для решения ряда фундаментальных задач, например, определения причин изменчивости зон распространения речных вод [1,2] или биологических объектов [3] при разных метеоусловиях.

Необходимым условием для такого рода расчётов является наличие поля скоростей на каждый момент времени вычислений. Большинство существующих в настоящее время методов расчета траекторий плавающих объектов использует в своей основе трехмерные гидродинамические модели различной сложности [4,5], в рамках которых результаты расчетов во многом зависят от адекватного описания скоростей течений используемыми моделями.

В настоящей работе предлагается метод расчета перемещения плавающих объектов, который базируется только на данных спутниковых измерений и атмосферных реанализов. На основе данного метода была создана система FOTS (Floating Object Tracking System) которая позволяет определять траектории плавающих объектов в любой точке Мирового океана. 


\section{Метод расчета}

Скорость частицы на поверхности можно определить из уравнения Навье-Стокса:

$$
\frac{d V}{d t}+\vec{m} \times f \vec{V}=-g \nabla h+\frac{1}{\rho} \frac{d \tau}{\partial z}
$$

Здесь V - скорость, $\tau$ напряжение трения ветра, $\mathrm{h}$ - уровень моря, $\rho$ плотность, $\mathrm{g}$ - ускорение свободного падения, $\mathrm{f}$ - параметр Кориолиса, $\mathrm{z}$ - глубина, $\vec{m}=\{-1,1,0\}$.

Первый член уравнения (1) описывает нелинейные члены в уравнение движения, которые можно считать малыми вдали от пограничных областей океана. Локальный градиент уровня моря считается не зависимым от напряжения трения ветра [6]. Тогда уравнение (1) можно разделить на два:

$$
\begin{aligned}
\vec{m} \times f \vec{V}_{g} & =-g \nabla h \\
\vec{m} \times f \vec{V}_{e} & =-\frac{1}{\rho} \frac{d \tau}{\partial z}
\end{aligned}
$$

Здесь $\vec{V}_{g}$ - поверхностная геострофическая скорость течений, $\vec{V}_{e}$ - дрейфовая скорость. Суммарная скорость поверхностных течений $V$ будет равна $V=\vec{V}_{g}+\vec{V}_{e}$.

Геострофическая скорость определяется по спутниковым альтиметрическим измерениям. Комбинированные измерения спутниковых альтиметров позволяют рассчитывать ежесуточные карты аномалий уровня моря на регулярной сетке с разрешением до 1/8 ${ }^{\circ}$ [7]. Полная динамическая топография $h$ определяется как сумма альтиметрических аномалий уровня моря и средней динамической топографии - специальной геофизической коррекции к данным альтиметров. Для глобального массива данных используется средняя динамическая топография из работы [8], для Черного моря из работы [9]. Геострофические скорости рассчитываются из уравнения (2) по градиентам динамической топографии.

Дрейфовая компонента течений $V_{e}$ была параметризована при сравнении с данными дрифтерных измерений , альтиметров и ветра в [10]. Дрейфовая компонента течений определялась как $V_{e}=0.028 \cdot V_{w} e^{\frac{i \pi}{36}}$, где $V_{w}$ - скорость ветра, т.е. скорость дрейфовых течений на поверхности составляла $2.8 \%$ от скорости ветра, а угол поворота поверхностных течений составил $13^{\circ}$ вправо. Для определения скорости ветра используются либо данные атмосферных реанализов NCEP ( могут быть использованы также данные MERRA, либо спутниковые измерения скатеррометров. Данные NCEP имеют разрешение 1 ○ и 6 часов (http://oceandata.sci.gsfc.nasa.gov/) данные MERRA имеют разрешение $0.5^{\circ *} 0.66^{\circ}$ и 6 часов (http://goldsmr2.sci.gsfc.nasa.gov/).

Полная поверхностная скорость течений определяется как векторная сумма $\mathrm{V}=\mathrm{V}_{\mathrm{g}}+\mathrm{V}_{\mathrm{e}}$. Пример поля геострофической скорости, поля скорости ветра и восстановленной карты полных поверхностных течений за 5 апреля 2016 12:00 изображен на рис. 1. Для восстановления лагранжевой траектории частиц по Эйлеровым полям скорости координаты виртуального объекта $\mathrm{r}^{\mathrm{n}}$ на шаге расчета $\mathrm{n}$ определялись по схеме Эйлера как:

$$
r_{n+1}=r_{n}+V\left(r_{n}\right) \cdot d t
$$

здесь $\mathrm{V}$-поверхностная скорость течений, линейно интерполированная на точку с координатами $\mathrm{xn}, \mathrm{yn} ; \mathrm{dt}$ - шаг расчетов по времени.

Для учета нелинейных членов, турбулентных процессов, ошибок задания параметров на каждом шаге времени частице задается дополнительное перемещение $d l$, пропорциональное скорости частицы:

$$
d l=P \cdot K_{T} \cdot d L
$$

здесь $P$ - случайная величина (от 0 до 1); значение $K_{T}=0.1$ является аналогом коэффициента турбулентной горизонтальной диффузии; $d L=r_{n+1}-r_{n}$ - перемещение частицы на данном шаге. 

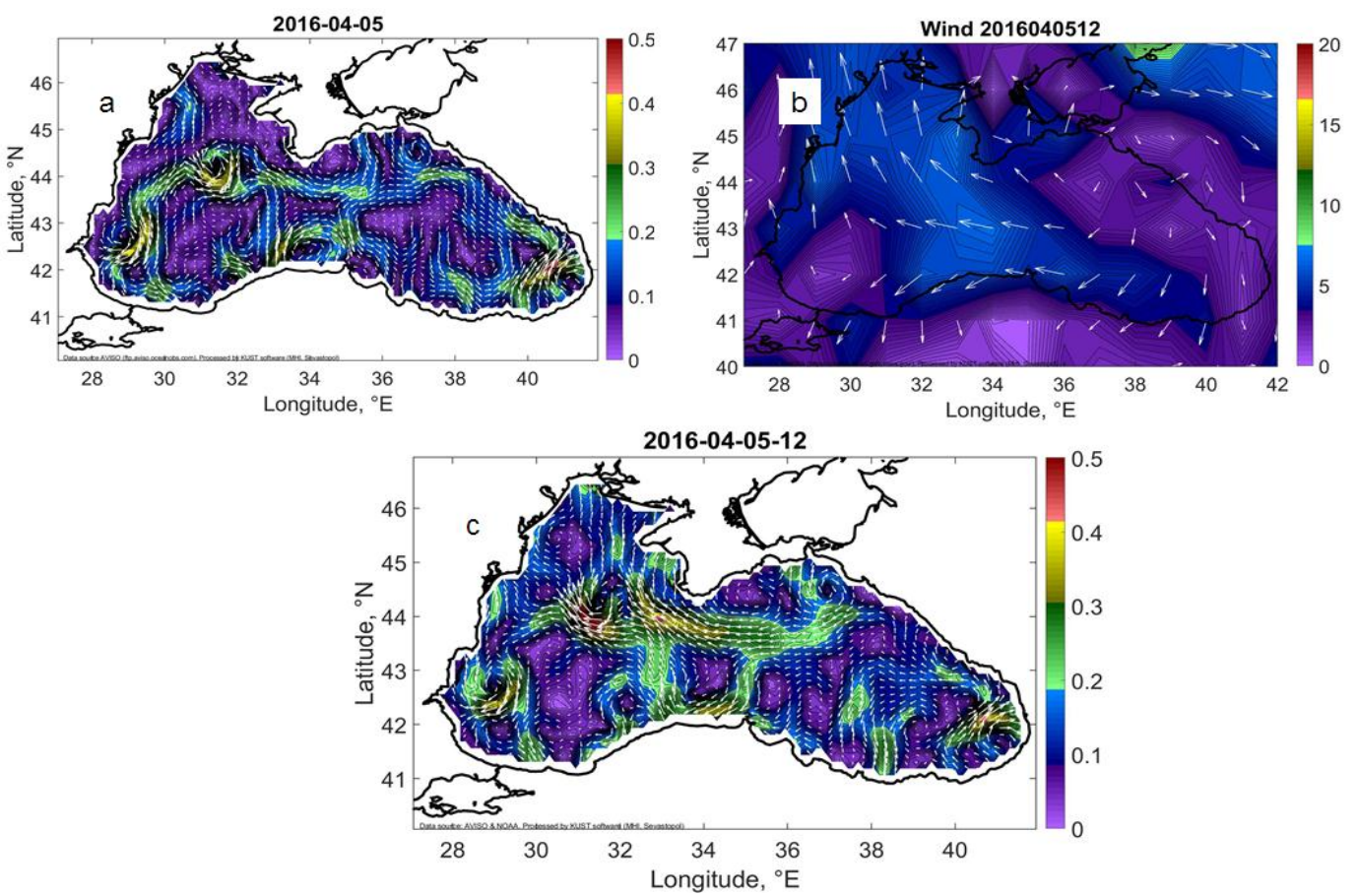

Рис. 1. Пример поля геострофической скорости (a), поля скорости ветра по данным NCEP (b) и восстановленной карты полных поверхностных течений (c) за 5 апреля 2016 12:00 UTC

\section{Примеры расчета перемещения плавающих объектов}

На рис. 1 представлен пример использования предложенного метода для расчетов перемещения нефтяной пленки, зафиксированной на двух последовательных радиолокационных снимках в Черном море. Заштрихованная область на рис. 2 представляет контур нефтяной пленки, наблюдавшийся на снимкеах радара SAR-C 03:48 UTC 23.05.2018 и зелёный контур соответствует границам слика в 15:43 UTC 23.05.2018. Результат расчета перемещения предлагаемым методом из точки центра области слика представлен на рис. 2 фиолетовой линией с учетом горизонтальной турбулентности. Предложенная модель успешно воспроизвела направление движения и расстояние на которое переместилось нефтяное пятно.

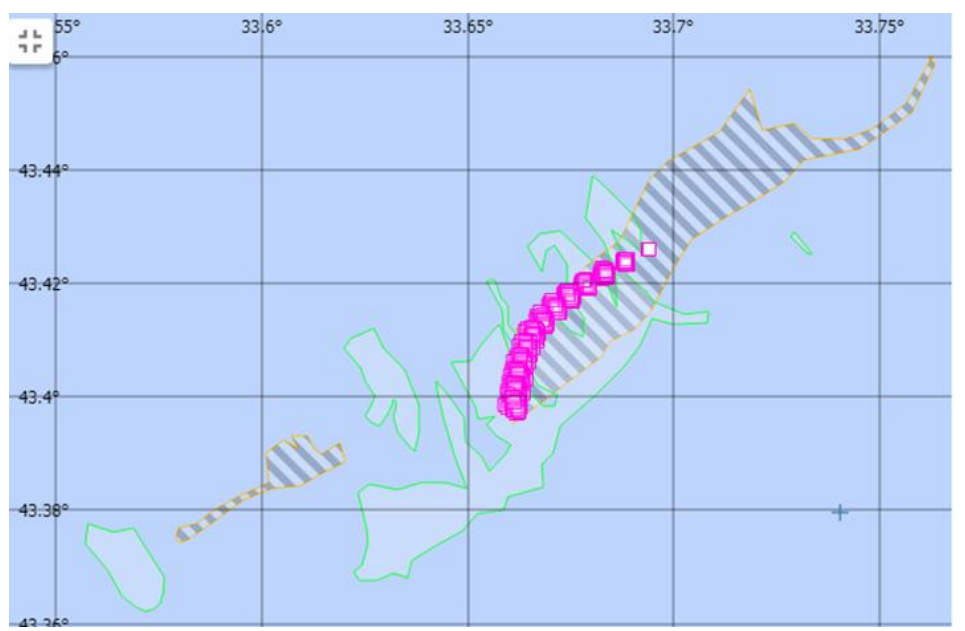

Рис.2. Смещение нефтяного пятна (заштрихованная область - зелёный контур) по данным последовательных радиолокационных наблюдений, наблюдавшийся на снимках

SAR-C 03:48 UTC 23.05.2018 Sentinel 1B и в 15:43 UTC 23.05.2018 Sentinel 1A соответственно и по результатам расчета (фиолетовые точки).

Еще один пример тестирования модели показан на рис. 3. Продолговатый слик необычной формы, вероятно вызванный разливом с проходящего судна, был зафиксирован в Средиземном море на снимке ASAR 26 сентября 2011 года. Такая форма пятна скорее всего связана с его смещением под действием поверхностных течений. Для моделирования 
перемещения этого слика задавался примерный курс проходящего судна (зеленая линия). Расчет перемещения нефти от заданного курса, представленный на рис.3 синей линией, хорошо совпадает с формой зарегистрированного слика. Таким образом, предлагаемый метод позволяет оценивать влияние мезомасштабных процессов на транспорт плавающих объектов. Отметим, что в этом случае проверка методики проведена с использованием единичного снимка и оценкой воспроизводимости трансформации пространственных структур.

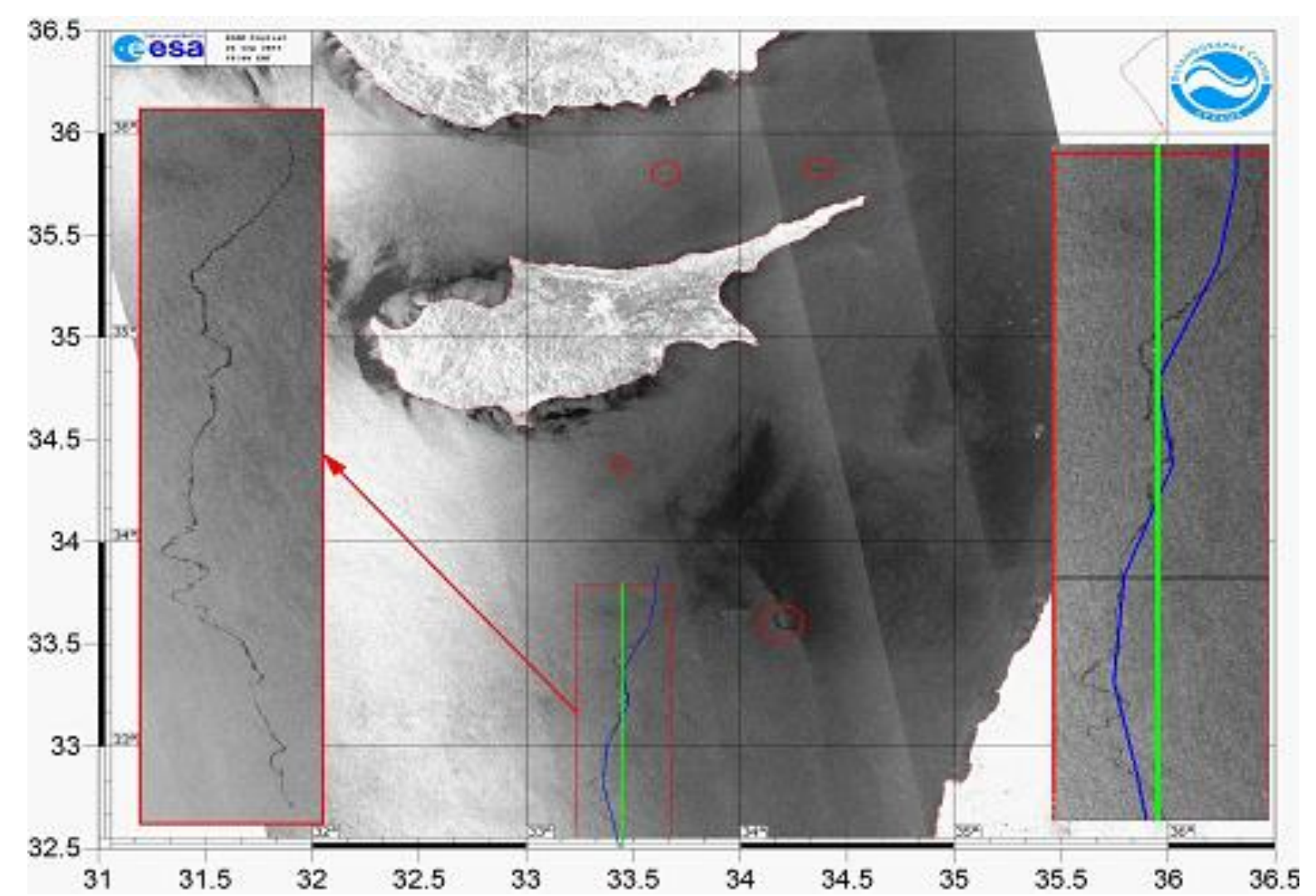

Рис.3. Радиолокационное изображение ASAR за 26 сентября 2011. Зеленая линия - примерный курс проходящего судна, синяя линяя - результаты расчета.

В ряде случаев предлагаемый подход может быть применён не только к плавающим объектам, но и к расчёту перемещения локальных особенностей в полях взвеси или температуры. Применение указанного метода для расчёта перемещения вихревой структуры, находящейся в зоне Основного Черноморского Течения хорошо регистрируемой на снимках сканера MODIS за 5 и 8 апреля 2016 года продемонстрировало адекватность предложенных расчётов для описания таких процессов. На рис 4 показаны карты сканера MODIS Aqua в «естественных» цветах для восточной части Черного моря за 5 апреля 2016 года (a) и 8 апреля 2016 года (b). Стрелками указано положение перемещающегося вихря с диаметром порядка 10-15 км, проявляющегося как область с увеличенным рассеянием за счёт аккумулируемой взвеси. На рис 4(с) красной линией показана расчётная траектория, жёлтые окружности соответствуют положениям вихря за 5 и 8 апреля соответственно. За трое суток вихрь сместился на 40 км в северо-западном направлении, что хорошо воспроизвелось модельными расчётами. 

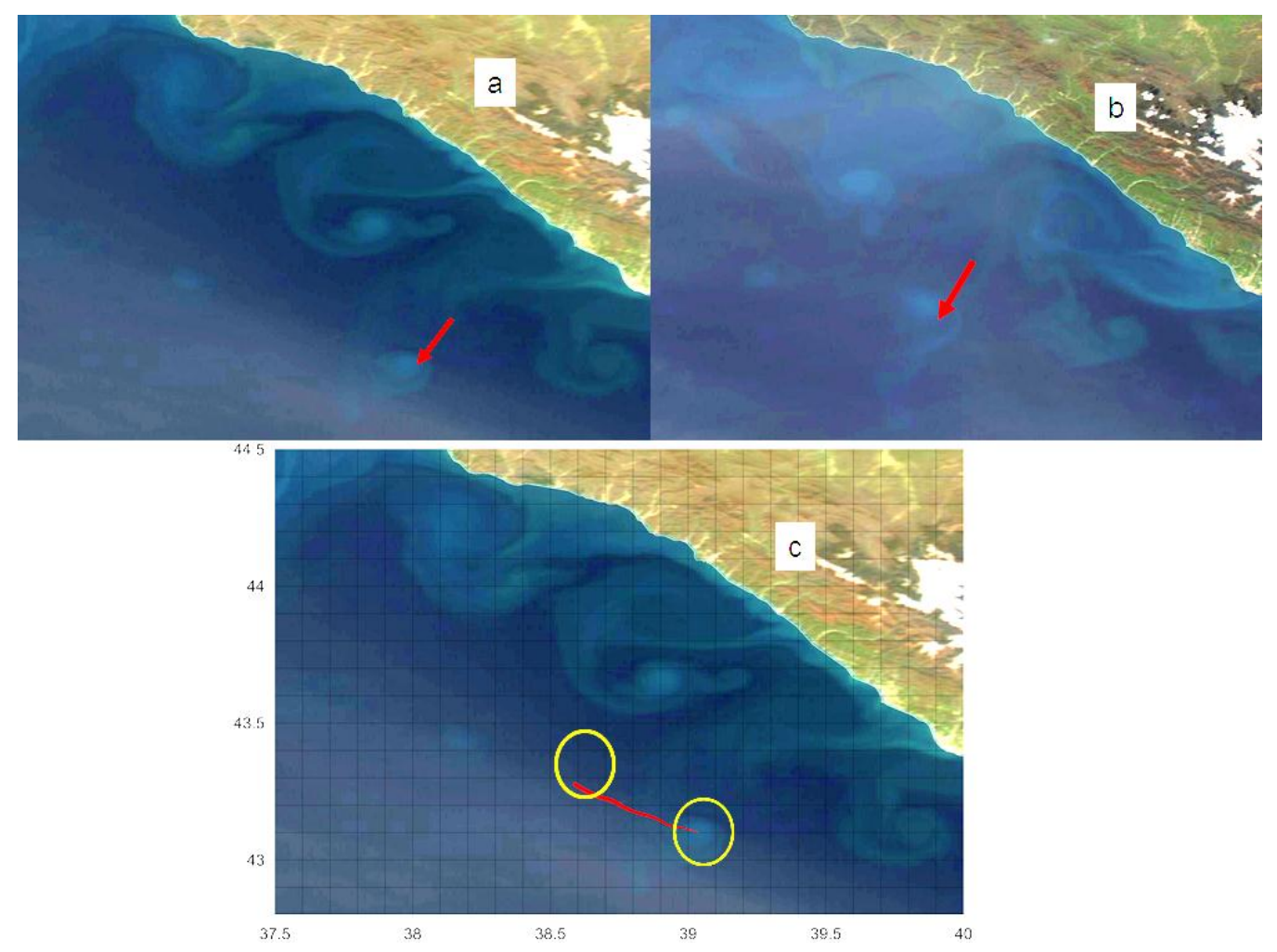

Рис. 4. Карты сканера MODIS в естественных цветах за 5 и 8 апреля 2016 года (a,b),

Расчётная траектория за трое суток (красная линия) и положения вихря за 5 и 8 апреля (жёлтые окружности)

В качестве предмета сравнения для оценки адекватности методики могут быть использованы не только отдельные траектории, но и области перемещения виртуальных частиц от известных источников. Подобного рода расчёты были проведены для Карского и Чёрного моря с целью исследования межгодовой изменчивости и определяющих её факторов $[11,12]$. На рис.5 представлен расчет распространения речных вод от устья Дуная в Черном море и его сопоставление с оптическими спутниковыми измерениями MODIS. Для расчета вблизи устья Дуная были установлены 4 постоянных источника виртуальных частиц. Рассчитанная концентрация виртуальных частиц (рис. 5 - слева) хорошо согласуется с зоной повышенных значений концентрации хлорофилла А. характерных для мутных и богатых биогенами шельфовых вод.
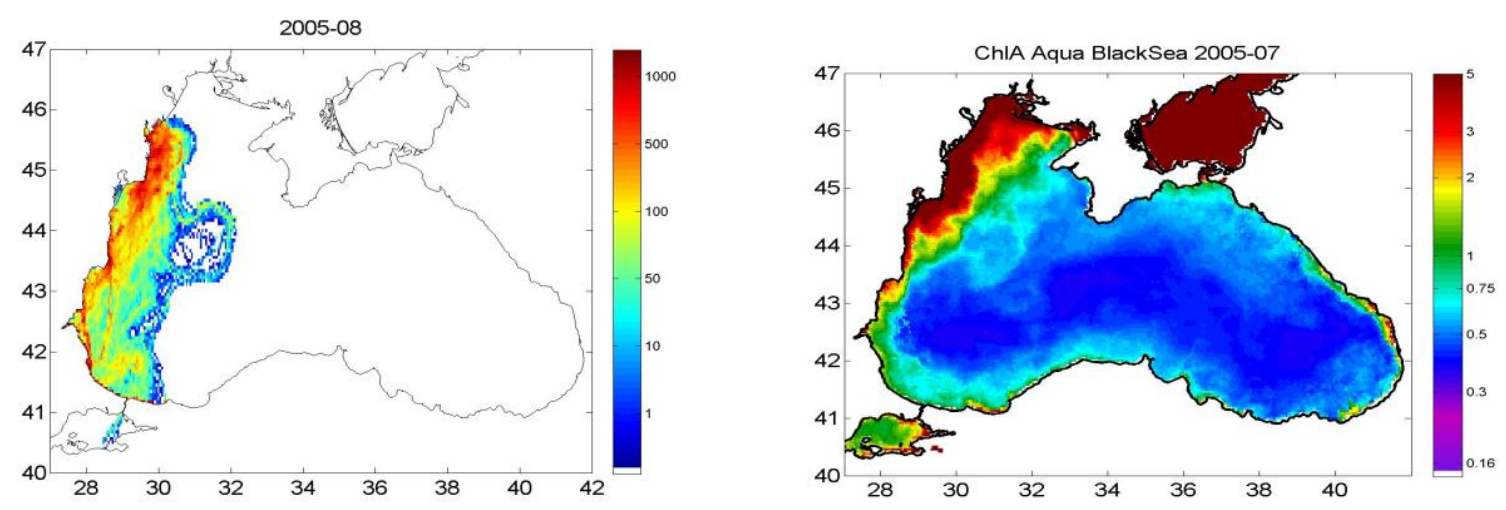

Рис. 5. Слева - Рассчитанная концентрация виртуальных трассеров, распространяющихся от устья Дуная в летний период 2005 г. в Черном море. Справа - средняя концентрация хлорофилла А в июле 2005 г. по данным MODIS 


\section{Комплекс расчета перемещения плавающих объектов FOTS}

На основе предложенного метода была создана система расчета перемещения плавающих объектов FOTS (floating object tracking system). Система позволяет рассчитывать не только распространение пассивных частиц в океане, но и распространение нефтяных загрязнений с учетом процессов, влияющих на их динамику и массообмен с окружающей средой (т.е. растекания, испарение и т.д.). Кроме того, при расчете траектории частиц в северных морях система учитывает ледовую обстановку в регионе.

Разработанная система позволяет рассчитывать перемещение точечного объекта (пример - единичный разлив загрязняющих веществ, обломки судна, льдина), группы объектов (разлив пятна нефти), распространение вещества от постоянного источника (авария на буровой платформе), как в прямом направлении с возможностью прогноза на 3 дня от текущего момента, так и в обратном направлении для определения источника загрязнений. Для учета различной парусности объекта в системе имеется возможность задавать дрейфовый коэффициент. Система представлена в виде отдельного приложения, которое легко устанавливается на персональный компьютер пользователя. Для работы системы требуется только подключение к Internet.

Пример расчёта направления распространения сточных вод с использованием системы FOTS для подводного канализационного выпуска в районе Севастополя приведён на рисунке 6 . Однако, для прибрежных районов (10-15 км от берега) применимость системы ограничена, что объясняется отсутствием альтиметрических данных вблизи берега и отличием реальной альтиметрии от экстраполированной за счёт существования мезомасштабных и субмезомасштабных процессов, а также недостаточным пространственным разрешением используемого ветрового форсинга. Успешными в прибрежной зоне для моделирования Севастопольского выпуска оказались только $40 \%$ ситуаций.

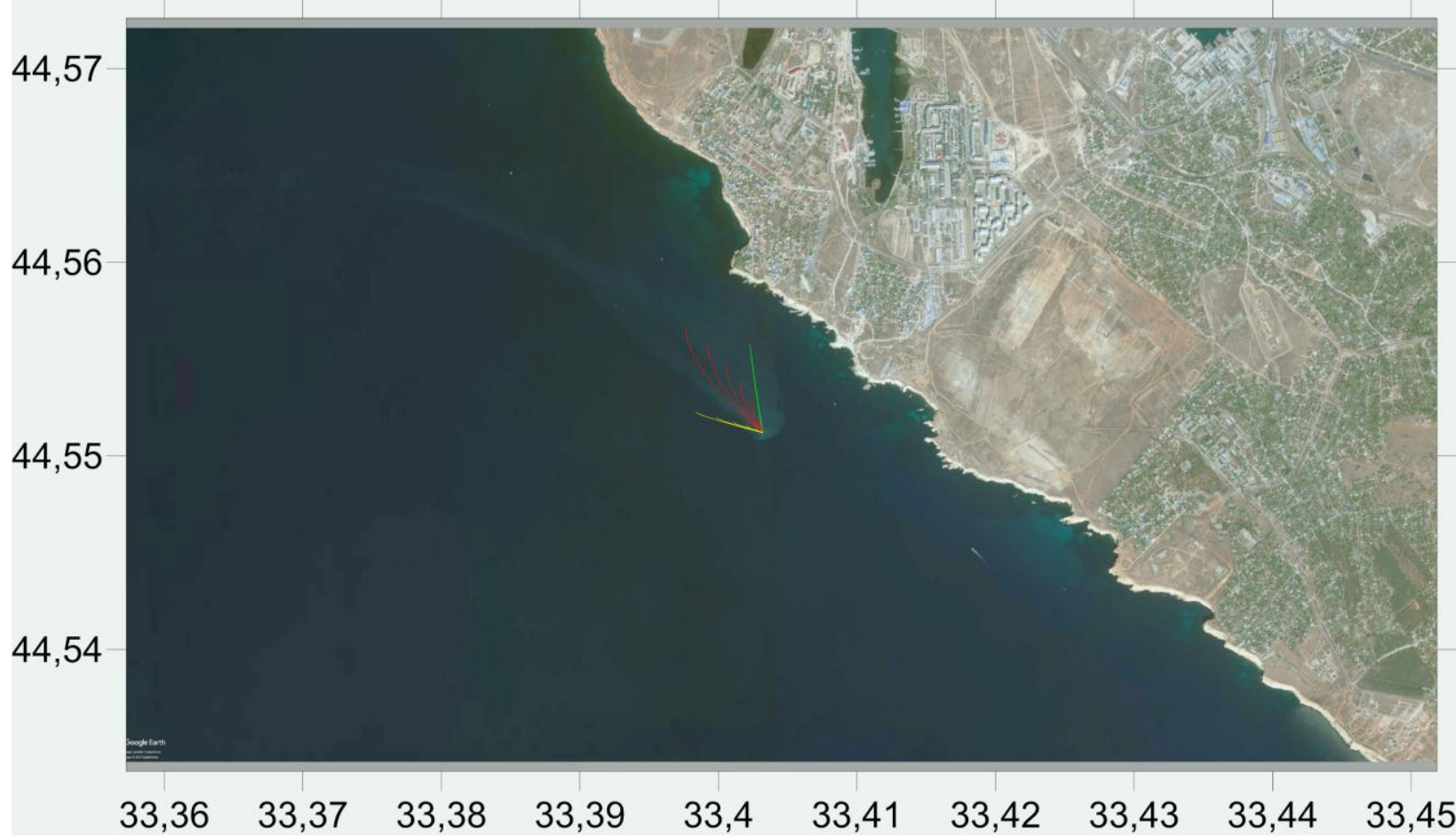

Рис. 6. Распространение загрязнений из подводного выпуска канализации вблизи Севастополя. Красными линиями расчёт из постоянного источника на 5 часов, жёлтые линии - только ветровой дрейф, зелёная линия геострофический дрейф. 


\section{Заключение}

В работе разработан метод, позволяющий на основе спутниковых альтиметрических измерений и данных о скорости ветра, рассчитывать перемещение плавающих объектов на поверхности моря. Метод был успешно опробован на основе сопоставления с данными радиолокационных и оптических снимков в различных морях Мирового океана. Расчёты в прибрежных зонах имеют большие ошибки, связанные с пространственно - временным разрешением альтиметрических данных и плохой воспроизводимостью скорости ветра. На основе предложенного метода в виде отдельного Windows приложения была создана система расчета перемещения плавающих объектов FOTS (floating object tracking system).

Работа выполнена при поддержке гранта РФФИ 18-45-920065 р_а в рамках госзадания, тема 0827-2019-0002.

\section{References}

[1]. Kubryakov A.A., Stanichny S.V., Zatsepin A.G. River plume dynamics in the Kara Sea from altimetrybased lagrangian model, satellite salinity and chlorophyll data, Remote Sensing Of Environment 176:177-187 . April 2016, DOI: 10.1016/j.rse.2016.01.020,

[2]. Kubryakov A.A., Stanichny S.V., Zatsepin A.G. Interannual variability of Danube water propagation during the summer from 1992 to 2015 and its influence on the Black Sea ecosystem, Journal of Marine Systems, 16 November 2017, ISSN 0924-7963, https://doi.org/10.1016/j.jmarsys.2017.11.001.

[3]. Rudorff, C. A. G., Lorenzzetti, J. A., Gherardi, D. F., \& Lins-Oliveira, J. E. Application of remote sensing to the study of the pelagic spiny lobster larval transport in the tropical Atlantic. Brazilian Journal of Oceanography, 2009, 57(1), 7-16.

[4]. Korotenko K.A, Mamedov R.M, Mooers C.N.K. Prediction of the dispersal of oil transport in the Caspian Sea resulting from a continuous release, Spill Science \& Technology Bulletin 6 (5-6), 323-339.

[5]. Kubryakov, A. I., Korotayev, G. K., Thoorens, F. X., Liungman, O., \& Ambjorn, C. New tool for the Black Sea environmental safety: BlackSea Track Web. In EGU General Assembly Conference Abstracts, 2012, Vol. 14, p. 4432.

[6]. Luther, D. S., Chave, A. D., Filloux, J. H., \& Spain, P. F. Evidence for local and nonlocal barotropic responses to atmospheric forcing during BEMPEX, Geophysical Research Letters, 1990, 17(7), pp. 949-952. [7]. Le Traon, P. Y., Dibarboure, G., \& Ducet, N. Use of a high-resolution model to analyze the mapping capabilities of multiple-altimeter missions, Journal of Atmospheric and Oceanic Technology, 2001, 18(7), pp.1277-1288.

[8]. Rio, M. H., Guinehut, S., \& Larnicol, G. New CNES CLS09 global mean dynamic topography computed from the combination of GRACE data, altimetry, and in situ measurements, Journal of Geophysical Research: Oceans, 2011, 116(C7).

[9]. Kubryakov, A.A., Stanichny, S.V. Mean Dynamic Topography of the Black Sea, computed from altimetry, drifter measurements and hydrology data, Ocean Science, 2011, 7 (6), pp. 745-753. DOI: 10.5194/os-7745-2011.

[10]. Stanichny, S.V., Kubryakov, A.A., Soloviev, D.M. Parameterization of surface wind-driven currents in the Black Sea using drifters, wind, and altimetry data, Ocean Dynamics, 2016, 66 (1), pp. 1-10. DOI: 10.1007/s10236-015-0901-3.

[11]. Kubryakov A.A., Stanichny S.V., Zatsepin A.G. Interannual variability of Danube water propagation during the summer from 1992 to 2015 and its influence on the Black Sea ecosystem, Journal of Marine Systems, 2017, https://doi.org/10.1016/j.jmarsys.2017.11.001.

[12]. Kubryakov A.A., Stanichny S.V., Zatsepin A.G. River plume dynamics in the Kara Sea from altimetrybased lagrangian model, satellite salinity and chlorophyll data, Remote Sensing Of Environment 176:177-187 .

(2016) · DOI: 10.1016/j.rse.2016.01.020. 